\title{
MAUS-TRATOS NA INFÂNCIA DE MULHERES VÍTIMAS DE VIOLÊNCIA
}

\author{
Maria Arleide da Silva \\ Gilliatt Hanois Falbo Neto \\ José Eulálio Cabral Filho"
}

\begin{abstract}
RESUMO. O presente estudo teve por objetivo identificar os comportamentos de risco de mulheres que presenciaram violência na família durante a sua infância e/ou adolescência e foram vítimas de violência na vida adulta, bem como determinar a prevalência desses casos e o perfil dessas mulheres. As mulheres investigadas eram assistidas no ambulatório de ginecologia do Centro de Atenção à Mulher do Instituto Materno-Infantil Professor Fernando Figueira, um centro de prestação de serviço terciário de saúde localizado no Nordeste do Brasil. O estudo foi um corte transversal e foram realizadas análises uni e bivariada em 619 questionários aplicados. Os resultados indicaram uma elevada prevalência $(39,7 \%)$ de história de violência familiar na infância e/ou adolescência de mulheres vítimas de violência na vida adulta. Concluiu-se que é importante e necessário identificar e assistir as famílias de risco, com o propósito de prevenir a prática de violência, haja vista a relevância da sua transmissão transgeracional.
\end{abstract}

Palavras-chave: violência, transmissão transgeracional, violência contra a mulher.

\section{MISTREATMENT IN CHILDHOOD AMONG FEMALE VICTIMS OF VIOLENCE}

\begin{abstract}
The aim of the present study was to determine the prevalence of women who had witnessed family violence in childhood and/or adolescence and were victims of violence in adult life, as well as identify the profile and risk behavior. The women investigated were being treated in a gynecology clinic at a tertiary healthcare service in northeastern Brazil. A cross-sectional cohort study was carried out, using univariate and bivariate analysis on 619 applied questionnaires. The results indicated a high prevalence of family violence in childhood and/or adolescence among female victims of violence in adult life (39.7\%). Conclusions were drawn regarding the importance and need for identification and assistance to families at risk in order to prevent the practice of violence, which has been demonstrated to have cross-generational transmission.
\end{abstract}

Key words: Violence, cross-generational transmission, violence against women.

\section{MALOS TRATOS EN LA NIÑEZ DE MUJERES VÍCTIMAS DE VIOLENCIA}

RESUMEN. El presente estudio tuvo por objetivo determinar la preponderancia, identificar el perfil y comportamientos de riesgo desarrollados por mujeres que han vivenciado violencia en la familia durante su niñez y/o juventud y han sido víctimas de violencia en la vida adulta. Las mujeres investigadas eran asistidas en el ambulatorio de ginecología de un servicio terciario de salud en Nordeste de Brasil. El estudio fue un corte transversal y se realizó un análisis uni y bivariada en 619 cuestionarios aplicados. Los resultados indicaron una alta preponderancia $39,7 \%$ de histórico de violencia familiar en la niñez y/o juventud de mujeres víctimas de violencia en la vida adulta. Se concluyó por la importancia y necesidad de identificación y asistencia a las familias de riesgo, con el propósito de prevenir la práctica de violencia dada la relevancia de la su transmisión transgeracional.

Palabras-clave: Violencia, transmisión transgeracional, violencia contra la mujer.

A violência contra a mulher é um grave e complexo problema de saúde pública. Os seus modelos causais evidenciam a existência de vários fatores associados, vinculados à sociedade, à comunidade, às relações interpessoais e ao perpetrador da violência (Heise, 1994; Reichenheim et al., 2006; García-Moreno, Jansen, Ellsberg, Heise \& Watts, 2006).

* Psicóloga, Psicanalista, Mestre e Doutoranda em Saúde Materno Infantil. Membro do Grupo de Estudos da Violência/Diretoria de Pesquisa/Instituto de Medicina Integral Prof. Fernando Figueira/IMIP.

\# Doutor em Medicina Materno Infantil. Coordenador do Grupo de Estudos da Violência/Diretoria de Pesquisa/IMIP.

II Pós-Doutor em Neurofarmacologia. Vice-coordenador do Programa de Pós-graduação/IMIP. 
Schraiber, D'Oliveira e Couto (2006), em revisão da literatura, concluíram que nos últimos cinco anos o tema ganhou dimensão social e ampliou-se o número de estudos realizados. Entretanto, devido à utilização de diferentes desenhos e instrumentos para a sua mensuração, ainda não se tem uma visão uniforme da real magnitude do problema. (Castro \& Riquer, 2003).

A distinção de gênero posta entre os atributos vinculados ao masculino e ao feminino fez desenvolver-se. cultural e socialmente. uma associação do primeiro com o homem, conferindo-lhe poder/dominação/força física entre outros, e do segundo com a mulher, atribuindo-lhe características como fragilidade e submissão. Há uma dessemelhança flagrante na relação de gênero entre homens e mulheres quando se observa o exercício da violência. Esta dissimilitude é marcada por ações agressivas do homem, baseadas na virilidade e consentidas socialmente, o contrário se verificando em relação à mulher, que apresenta uma atitude de subserviência e aceitação da primazia masculina (Saffiotti, 1999).

A Pós-Modernidade não eliminou as dessemelhanças de gênero quanto às condições de homens e mulheres na sociedade. Embora a mulher tenha realizado grandes conquistas sociais, a discriminação de gênero coloca-a ainda numa condição de inferioridade na comparação com o homem. Saffiotti refere-se ao assassinato de mulheres na contemporaneidade como um simile do poder do patriarca na Roma Antiga, que podia arbitrar sobre a vida e a morte de sua mulher (Saffiotti, 2005).

As práticas violentas contra a mulher ocorrem mais frequientemente no ambiente restrito do lar, fato que reduz a sua visibilidade e o seu registro (Cavalcanti, 2003). Entre as várias consequiências da violência contra a saúde da mulher podem-se destacar efeitos mortais - como assassinato, suicídio, mortalidade materna e efeitos relacionados com a Aids; e efeitos que não são mortais, mas afetam a saúde física, como lesões, alterações funcionais, incapacidade permanente e transtornos crônicos. No tocante à saúde mental, identificam-se o estresse pós-traumático, depressão, angústia, fobias, transtorno do pânico, transtornos alimentares, disfunção sexual, baixa auto-estima e abuso de substâncias psicoativas (Pan American Health Organization, 2003).

A situação de risco como consequiência da violência estende-se aos filhos das mulheres vitimadas. Presenciar violência conjugal aumenta nas crianças a probabilidade de sofrer depressão, ansiedade, transtornos de conduta e atrasos no desenvolvimento cognitivo (Fantuzzo, citado por Klevens, 2001).

Segundo Lamanno-Adamo (1999), a violência é adotada como estratégia de sobrevivência pelo fato de ser reconhecida como um "bem", e não como um dano, motivo pelo qual ela se mantém e se recria.
Nos últimos anos, os estudos de violência contra a mulher, indistintamente dos desenhos adotados e das localidades onde os mesmos se realizem, têm encontrado elevadas prevalências de violência física, sexual e/ou verbal (Deslandes, Gomes \& Silva, 2000; Silva, 2003; Reichenheim et al., 2006; García-Moreno et al., 2006). A magnitude do problema e a recomendação da OMS para que se incrementassem os estudos a fim de se conhecer mais fielmente a dimensão desse problema geraram um grande número de estudos de prevalência. Embora esses mantenham a sua importância - inclusive pela condição de serem um marco inicial para o estudo de problemas desta natureza - e produzam o conhecimento necessário, é mister o seu aprofundamento, de modo que se possam identificar as causas e gerar assim a prevenção possível.

No Brasil, a violência contra a mulher ocupa hoje um lugar de destaque, o que a coloca como um dos problemas prioritários na saúde pública (García-Moreno, 2000; World Health Organization, 2001). Assim, a realização de estudos para investigar a magnitude do problema e ampliar o conhecimento das suas causas pode fornecer subsídios para políticas públicas que abranjam a saúde da mulher na sua integralidade. Ademais, a transmissão transgeracional tem sido apontada como um dos fatores importantes para o crescimento da violência. (Klevens, 2001; Vizcarra, Cortés, Bustos, Alarcón \& Muñoz, 2001). Neste sentido, a realização deste estudo torna-se relevante pela oportunidade de contribuir para o conhecimento dos motivos que promovem o recrudescimento da violência, com ênfase na transmissão da violência entre gerações.

\section{MÉTODO}

Esta pesquisa é parte de um estudo mais amplo, do tipo corte transversal, que procurou estimar a prevalência de violência contra a mulher com história de ter presenciado violência familiar na infância e/ou adolescência, em mulheres assistidas no Ambulatório de Ginecologia do Centro de Atenção à Mulher/Instituto Materno Infantil Professor Fernando Figueira (CAM/IMIP), no período de outubro/2005 a janeiro/2006.

O tamanho da amostra calculado para o estudo original foi de 532 sujeitos. Aceitando-se um erro de estimação não superior a $3 \%$ e um nível de confiança de 95\% e adotando-se procedimento de amostragem sistemática, este valor foi ampliado para 619, para prevenir possíveis perdas. No segmento desse estudo que corresponde à presente pesquisa foram incluídas mulheres com idade igual ou superior a 19 anos, não grávidas, que presenciaram violência familiar na infância e/ou adolescência e sofreram violência na vida adulta. Foram excluídas mulheres que já haviam respondido a esta 
pesquisa e mulheres gestantes, resultando em uma amostra de 170 participantes.

O objetivo principal deste estudo foi identificar mulheres que foram vítimas de violência nos últimos 12 meses e presenciaram violência em sua infância e/ou adolescência. Assim, as análises realizadas tomaram como referência o percentual de mulheres investigadas que nas suas declarações atenderam ao objetivo proposto.

Para a coleta dos dados foi utilizado o instrumento Abuse Assessment Screen (AAS) (Reichenheim, Moraes \& Hasselmann, 2000), adaptado mediante a retirada da pergunta sobre violência na gestação e acrescido de questões fechadas, incluindo fatores econômicos e sociodemográficos, história de violência familiar na infância e/ou adolescência da mulher, uso de bebidas alcoólicas e outras drogas. Três auxiliares de pesquisa foram treinados para a realização do estudo-piloto e coleta de dados. Para o treinamento utilizou-se um manual de instruções confeccionado exclusivamente para este fim. A ênfase do manual foi destacar a importância do estudo, a questão ética e a necessidade de um acolhimento eficaz, com encaminhamento das vítimas identificadas a um serviço especializado, garantindo atendimento psicológico ou médico de urgência no decorrer da aplicação do questionário, se houvesse necessidade.

As entrevistas foram realizadas em sala reservada, no Ambulatório da Mulher do IMIP, garantindo a confidencialidade e o sigilo necessários a procedimentos desta natureza. excluídos.

Houve apenas uma recusa, e 11 questionários foram

As respostas foram digitadas em um banco de dados com dupla entrada, no programa EPI-INFO 6.04 d (CDC, Atlanta), tendo sido realizados testes de consistência das variáveis, com o objetivo de checagem e correção de possíveis erros.

O estudo obteve a aprovação do Comitê de Ética em Pesquisa em Seres Humanos do Instituto Materno Infantil Professor Fernando Figueira/IMIP.

\section{RESULTADOS}

O perfil sociodemográfico e econômico das 170 mulheres vítimas de violência (Tabela 1) evidenciou que a maioria $(53,5 \%)$ era procedente da região metropolitana do Recife, enquanto 38,8\% eram da própria cidade do Recife. A cor não-branca predominou em 80,6\%; 74,7\% encontravam-se abaixo da sexta década de vida e $42,4 \%$ declararam ter cursado o Ensino Fundamental. Do total das mulheres, $84,7 \%$ declararam ter companheiro durante o último ano e 45,9\% exerciam trabalho remunerado, embora apenas $15,4 \%$ tenham informado exercer trabalho com carteira assinada.
Tabela 1. Distribuição de Freqüência de Mulheres Vítimas de Violência com História de Violência Familiar na Infância/Adolescência Conforme Fatores Sociodemográficos e ECONÔMICOS, Atendidas no Período de Outubro/2005 a Janeiro/2006. CAM-IMIP.

\begin{tabular}{|c|c|c|c|c|c|c|c|c|}
\hline \multirow{3}{*}{$\begin{array}{c}\text { Fatores } \\
\text { sociodemográficos } e \\
\text { econômicos }\end{array}$} & \multicolumn{6}{|c|}{$\begin{array}{c}\begin{array}{c}\text { História de violência nainfância e } \\
\text { adolescência }\end{array} \\
\end{array}$} & \multirow{3}{*}{$X^{2} ; \mathbf{g l}$} & \multirow{3}{*}{ p valor } \\
\hline & \multicolumn{2}{|c|}{ Sim } & \multicolumn{2}{|c|}{ Não } & \multirow[b]{2}{*}{ Total } & \multirow[b]{2}{*}{$\%$} & & \\
\hline & $\mathbf{N}$ & $\%$ & $\mathbf{N}$ & $\%$ & & & & \\
\hline Procedência & & & & & & & $1.47 ; \mathrm{gl}=2$ & 20,478 \\
\hline Recife & 31 & 47,0 & 35 & 53,0 & 66 & 38,8 & & \\
\hline$R M R$ & 49 & 53,8 & 42 & 46,2 & 91 & 53,5 & & \\
\hline Interior do Estado & 5 & 38,5 & 8 & 61,5 & 13 & 7,6 & & \\
\hline Idade & & & & & & & $4.28 ; \mathrm{gl}=4$ & 0,368 \\
\hline$\geq 19$ a 29 anos & 18 & 47,4 & 20 & 52,6 & 38 & 22,4 & & \\
\hline 30 a 39 anos & 19 & 59,4 & 13 & 40,6 & 32 & 18,8 & & \\
\hline 40 a 49 anos & 30 & 52,6 & 27 & 47,4 & 57 & 33,5 & & \\
\hline 50 a 59 anos & 16 & 47,1 & 18 & 52,9 & 34 & 20,0 & & \\
\hline$>60$ anos & 2 & 22,2 & 7 & 77,8 & 9 & 5,3 & & \\
\hline Cor & & & & & & & $0.04 ; g l=1$ & 0,846 \\
\hline Branca & 16 & 48,5 & 17 & 51,5 & 33 & 19,4 & & \\
\hline Não-branca & 69 & 50,4 & 68 & 49,6 & 137 & 80,6 & & \\
\hline Escolaridade & & & & & & & 7.38; $\mathrm{gl}=3$ & 0,061 \\
\hline Não mstudou & 5 & 23,8 & 16 & 76,2 & 21 & 12,4 & & \\
\hline Ensino fundamental & 37 & 51,4 & 35 & 48,6 & 72 & 42,3 & & \\
\hline Ensino médio & 27 & 52,9 & 24 & 47,1 & 51 & 30,0 & & \\
\hline Superior & 16 & 61,5 & 10 & 38,5 & 26 & 15,3 & & \\
\hline Situação marital & & & & & & & $1.63 ; \mathrm{gl}=1$ & 0,201 \\
\hline Com companheiro & 75 & 52,1 & 69 & 47,9 & 144 & 84,7 & & \\
\hline Sem companheiro & 10 & 38,5 & 16 & 61,5 & 26 & 15,3 & & \\
\hline Trabalho remunerado & & & & & & & $0.00 ; \mathrm{gl}=1$ & 1,000 \\
\hline Sim & 39 & 50,0 & 39 & 50,0 & 78 & 4509 & & \\
\hline Não & 46 & 50,0 & 46 & 50,0 & 92 & 54,1 & & \\
\hline Filhos & & & & & & & $0.37 ; \mathrm{gl}=4$ & 0,540 \\
\hline Sim & 69 & 48,9 & 72 & 51,1 & 141 & 82,9 & & \\
\hline Não & 16 & 55,2 & 13 & 44,8 & 29 & 17,1 & & \\
\hline Renda familiar (SM) & & & & & & & $0.23 ; \mathrm{gl}=1$ & 0,634 \\
\hline Até 1 salário-mínimo & 28 & 47,5 & 31 & 52,5 & 59 & 34,7 & & \\
\hline Mais de 1 até 2 salários & 30 & 50,8 & 29 & 49,2 & 59 & 34,7 & & \\
\hline Mais de $2 \mathrm{SM}$ & 27 & 51,9 & 25 & 48,1 & 52 & 30,6 & & \\
\hline
\end{tabular}

Quanto aos tipos de agressão presenciados na infância e/ou adolescência (Tabela 2), encontrou-se que: $42,8 \%$ presenciaram xingamentos; 35,5\%, bofetadas; 33,1\%, ameaças com palavras; 28,3\%, espancamento; e 9,6\%, ameaças com armas. Do total de mulheres com história de violência na infância e/ou adolescência, $60,0 \%$ declararam o pai como perpetrador de maus-tratos no lar.

Tabela 2. Distribuição de Frequiência de Mulheres Vítimas de Violência Conforme os Tipos de Agressão Presenciada na Infância e/ou Adolescência, Atendidas no Período de Outubro/2005 a Janeiro/2006. CAM-IMIP.

\begin{tabular}{llc}
\hline Tipo de agressão presenciada \# & N & \% \\
\hline Xingamentos & 71 & 42,8 \\
Bofetadas & 59 & 35,5 \\
Ameaças com palavras & 55 & 33,1 \\
Espancamento & 47 & 28,3 \\
Ameaças com armas & 16 & 9,6 \\
\hline
\end{tabular}

\# Não excludentes

Fonte: CAM-IMIP/2006 
Neste estudo, dentre as 170 mulheres vítimas de violência, $39,7 \%$ tinham presenciado violência também na infância e/ou adolescência (Tabela 3). O resultado da análise da associação entre ter presenciado história de violência familiar na infância e/ou adolescência e sofrer violência na vida adulta nos últimos doze meses no período da coleta de dados foi significante $(p<0,001)$.

Tabela 3. Distribuição de Frequiência de Mulheres Vítimas de Violência Conforme História de Violência Familiar na Infância e/ou Adolescência, Atendidas no Período de Outubro/2005 a Janeiro/2006. CAM-IMIP.

\begin{tabular}{|c|c|c|c|c|c|c|c|c|}
\hline \multirow{3}{*}{$\begin{array}{l}\text { Presenciou violência familiar na } \\
\text { infância e/ou adolescência }\end{array}$} & \multicolumn{6}{|c|}{ Violência contra a mulher } & \multirow[b]{3}{*}{$X 2$; gl } & \multirow[b]{3}{*}{ p valor } \\
\hline & \multicolumn{2}{|c|}{ Sim } & \multicolumn{2}{|c|}{ Não } & \multirow[b]{2}{*}{ Total } & \multirow[b]{2}{*}{$\%$} & & \\
\hline & $\mathbf{N}$ & $\%$ & $\mathbf{N}$ & $\%$ & & & & \\
\hline Sim & 85 & 39,7 & 129 & 60,3 & 214 & 34,6 & 24.66; $\mathrm{gl}=1$ & $<0,01 *$ \\
\hline Não & 85 & 21,0 & 320 & 79,0 & 405 & 65,4 & & \\
\hline Total & 170 & 27,5 & 449 & 72,5 & 619 & 100 & & \\
\hline
\end{tabular}

$* \mathrm{p}<0,05$ significativo

Fonte: CAM - IMIP/2006

Os resultados da associação entre ter presenciado violência na infância e/ou adolescência e comportamentos de risco (Tabela 4) não foram significantes para o uso de outras drogas (categorizado em uso de comprimidos, drogas injetáveis, crack, maconha ou cocaína) e tabagismo. Houve associação positiva com uso de bebida alcoólica e significância estatística $(p=0,033)$ na investigação do padrão do uso de bebida alcoólica pelas mulheres deste estudo.

Tabela 4. Distribuição de Freqüência de Mulheres Vítimas de Violência que Presenciaram Violência Familiar na Infância e/ou Adolescência Conforme Comportamentos de Risco, Atendidas no Período de Outubro/2005 a Janeiro/2006. CAM-IMIP.

\begin{tabular}{|c|c|c|c|c|c|c|c|}
\hline \multirow[t]{3}{*}{ Comportamentos de risco } & \multicolumn{6}{|c|}{ Presenciar violência na infância e/ou adolescência } & \multirow[b]{3}{*}{ Exato de Fisher } \\
\hline & \multicolumn{2}{|c|}{ Sim } & \multicolumn{2}{|c|}{ Não } & \multirow[b]{2}{*}{ Total } & \multirow[b]{2}{*}{$\%$} & \\
\hline & $\mathbf{N}$ & $\%$ & $\mathbf{N}$ & $\%$ & & & \\
\hline Uso de bebida alcoólica & & & & & & & $\mathbf{0 , 0 2 7}$ \\
\hline Sim & 28 & 62,2 & 17 & 37,8 & 45 & 26,5 & \\
\hline Não & 57 & 45,6 & 68 & 54,4 & 125 & 73,5 & \\
\hline Bebe socialmente & 24 & 58,5 & 17 & 41,5 & 41 & 24,1 & \\
\hline Bebe uma vez por semana & 7 & 87,5 & 1 & 12,5 & 8 & 4,7 & \\
\hline Bebe duas vezes por semana & 0 & 0,0 & 2 & 100,0 & 2 & 1,2 & \\
\hline Não bebe & 54 & 45,4 & 65 & 54,6 & 119 & 70,0 & \\
\hline Uso de outras drogas \# & & & & & & & 0,368 \\
\hline Sim & 1 & 20,0 & 4 & 80,0 & 5 & 2,9 & \\
\hline Não & 84 & 50,9 & 81 & 49,1 & 165 & 97,1 & \\
\hline Tabagismo & & & & & & & Valor $p \mathbf{0 , 5 9 9 *}$ \\
\hline Sim & 7 & 43,8 & 9 & 56,3 & 16 & 9,4 & \\
\hline Não & 78 & 50,6 & 76 & 49,4 & 154 & 90,6 & \\
\hline
\end{tabular}

\# Comprimidos, drogas injetáveis, crack, maconha ou cocaína

$* X^{2} 0.28 ; \mathrm{gl}=1$

$* \mathrm{p}<0,05$ significativo

Fonte: CAM-IMIP/2006

\section{DISCUSSÃO}

No presente estudo, a elevada freqüência de presenciar violência na infância e/ou adolescência por parte de mulheres adultas vítimas de violência corrobora os resultados de Rivera-Rivera et al. (2004), que investigaram a violência contra a mulher e a transmissão da violência entre gerações.

Mulheres que reportam terem sido vítimas de violência durante a infância são mais susceptíveis de vivenciar agressões por parte de seus parceiros atuais. Houve, no presente estudo, um indicativo de que o baixo grau de escolaridade da mulher pode contribuir 
como fator associado para sofrer violência doméstica. Tais resultados são suportados pelo estudo realizado por Rivera-Rivera et al. (2004), que mostraram ser a violência contra a mulher inversamente proporcional ao grau de escolaridade. Indivíduos com menor escolaridade encontram-se mais frequientemente nas faixas populacionais em condições de pobreza, o que favorece a exclusão social e baixa auto-estima, reduzindo as perspectivas de que possam exercer atividades bem remuneradas no mercado de trabalho.

É relevante destacar que as violências intrafamiliares e contra a mulher ocorrem de forma indistinta e transversal na sociedade, além de comungarem o fato de acontecerem, com maior frequiência, no espaço privado da família. Esta constatação leva-nos a duas dificuldades na realização de pesquisas. A primeira alude às populações investigadas nos estudos sobre violência, os quais, quase por excelência, envolvem populações usuárias de serviços públicos - no caso do Brasil, aquelas que utilizam o Sistema Único de Saúde, onde é mais provável identificar pessoas de baixa condição social; a segunda diz respeito ao "pacto de silêncio", pela não-denúncia entre os familiares, como se precisassem proteger a relação dominador/dominado, na qual a pessoa vitimizada assume uma condição de subserviência em relação ao seu agressor. Esta última dificuldade representa uma limitação do nosso estudo, visto que pode estar presente também em classes socioeconômicas mais favorecidas, impedindo inferências comparativas entre populações de níveis sociais distintos.

Semelhantes aos nossos resultados foram os de Vizcarra et al. (2001), que encontraram 68,0\% de violência psicológica e $25,0 \%$ de violência física na vida conjugal de mulheres que declararam ter presenciado violência familiar na infância. Os autores investigaram também experiência de maus-tratos na infância, com os seguintes resultados: em 53,4\% dos casos, violência psicológica; em $52,7 \%$; violência física, e em 39,1\%, violência sexual. Menezes, Amorim, Santos e Faúndes (2003) encontraram história de violência na família da mulher como fator fortemente associado a sofrer violência física doméstica no puerpério, em se tratando de mulheres assistidas no IMIP/ Recife/Pernambuco.

Embora não possam ser generalizados, os presentes resultados suportam estudos psicanalíticos que têm ressaltado a importância da transmissão transgeracional da violência (Correa, 2003; Benghozi, 2005). Segundo Gomes (2005), a subjetividade, no contexto das relações conjugais e na estruturação familiar, utiliza o mecanismo de repetição, que faz parte do processo de elaboração ou fixação de uma situação traumática.

Permanecendo nesta ótica, a perspectiva psicanalítica defende que a estruturação do psiquismo humano implica um longo processo de construção em que o indivíduo lança mão de modelos, não havendo nesta escolha um juízo de valor. Esse fato concorre para, pelo processo de identificação, o indivíduo reproduzir também a violência (Freud, 1921/1974). A identificação (Freud, 1921/1974) é o laço afetivo que une o indivíduo aos seus pares, promove a cultura, faz sobreviver a instituição familiar e, ao longo da história, é transmitida de geração em geração, como forma de perpetuar a espécie humana. Conforme esta teoria, é na relação com a família ou com a pessoa que cuida que esses modelos se estabelecem e são reproduzidos. De acordo com Freud (1913/1974) em "Totem e Tabu", após os filhos da horda primeva assassinarem o pai tirano, foi necessário criarem um totem a quem venerar e prestar obediência, sendo esta a prerrogativa da lei para garantir a existência da espécie.

Assim, presenciar ou sofrer violência na infância pode resultar, na vida adulta, na aceitação de sofrer violência ou praticá-la como conduta comum e adequada. Conforme o modelo de identificação freudiano, seria a reprodução inconsciente de se repetir, na vida adulta, a violência presenciada ou vivida nas relações infantis (Freud, 1923/1974).

As elevadas prevalências de violência familiar presenciada ou sofrida na infância denunciam a vulnerabilidade do gênero, apontam para a violência contra a mulher em sua infância ou adolescência e reforçam a hipótese do uso da violência como padrão de conduta para a resolução de conflitos. Permitem também constatar a repetição do vivido e contribuem para a disseminação da violência e sua perpetuação.

A violência presenciada ou sofrida na infância ou adolescência tem uma importância fundamental na estruturação do psiquismo humano. Este registro mental pode abrir mais chances, na vida adulta, para a repetição de comportamentos violentos, pela identificação seja com o agressor seja com a vítima, como também pela utilização de tais comportamentos como forma de responder aos conflitos ou se relacionar no cotidiano (Correa, 2003; Benghozi, 2005). Ademais, as experiências traumáticas de violência sofridas e presenciadas na infância têm uma forte relação com práticas criminais de jovens adolescentes (Messina \& Grella, 2006).

A violência pode assumir o papel de mediadora das relações interpessoais. É o que afirmam Melo et al. (2007) em estudo realizado com adolescentes e 
jovens do Aglomerado Morro das Pedras, em Belo Horizonte. Um dos adolescentes, referindo-se às práticas violentas, diz que elas: “... Passa de pai para filho...". Talvez essa seja a maneira mais simples de expressar a forma como a violência se reproduz (Melo et al, 2007).

Os tipos de violência presenciados pelas mulheres variaram desde os xingamentos até ameaças com armas. A prática cotidiana de maus-tratos dentro da família aponta, entre outras questões relevantes, a pouca condição de resiliência familiar. As várias formas de violência, sejam humilhações verbais ou agressões com risco de morte, tornam-se registros de experiências psíquicas que, não sendo elaboradas, transformam-se em um terreno fértil para o adoecer psíquico.

O uso e padrão de uso de bebida alcoólica entre as mulheres deste estudo evidenciaram uma associação positiva com presenciar violência na infância e/ou adolescência e com sofrer violência na vida adulta. $\mathrm{O}$ uso de álcool pela mulher tem sido citado, em vários estudos, principalmente como conseqüência de ela sofrer violência; entretanto, recomenda-se considerar a hipótese de esse uso constituir-se em um dos fatores desencadeantes para ser vítima ou praticar violência (Velzeboer, Ellsberg, Clavel Arcas \& García-Moreno, 2003).

A realização de novas investigações, não apenas da violência contra a mulher, mas da sua transmissão transgeracional, abre possibilidades para uma prevenção mais eficaz. Ademais, este é fator causal de grande importância para a vitimização e para práticas violentas.

\section{REFERÊNCIAS}

Benghozi, P. (2005). Resiliência familiar e conjugal numa perspectiva psicanalítica dos laços. Psicologia Clínica, 17(2), 101-109.

Castro, R., \& Riquer, F. (2003). La investigación sobre violencia contra las mujeres en América Latina: Entre el empirismo ciego y la teoria sin datos. Cadernos de Saúde Pública, 19(1), 135-146.

Cavalcanti, A. L. (2003). Violência contra a mulher: um problema de saúde. Revista Ginecologia \& Obstetrícia, 14(4), 182-188.

Correa, O. B. R. (2003). Transmissão psíquica entre gerações. Psicologia, USP, 14(3), 35-450.

Deslandes, S. F., Gomes R., \& Silva C. M. F. P. (2000). Caracterização dos casos de violência doméstica contra a mulher atendidos em dois hospitais públicos do Rio de Janeiro. Cadernos de Saúde Pública, 16(1), 129-137.

Freud, S. (1974). Totem e Tabu. Edição Standard Brasileira das Obras Psicológicas Completas (J. Salomão, Trad., Vol. XIII, pp. 193207). Rio de Janeiro: Imago (Original publicado em 1913).

Freud, S. (1974). Recordar, repetir e elaborar. Edição Standard Brasileira das Obras Psicológicas Completas (J. Salomão, Trad.,
Vol. XII, pp. 193-207). Rio de Janeiro: Imago (Original publicado em 1914).

Freud, S. (1974). Psicologia de grupo e análise do ego. Edição Standard Brasileira das Obras Psicológicas Completas (J. Salomão, Trad., Vol. XVIII, pp. 89-179). Rio de Janeiro: Imago (Original publicado em 1921).

Freud, S. (1974). O ego e o id. Edição Standard Brasileira das Obras Psicológicas Completas (J. Salomão, Trad., Vol. XIX, pp. 13-83). Rio de Janeiro: Imago (Original publicado em 1923).

García-Moreno, C. (2000). Violencia contra la mujer: género y equidad en la salud/Violence against women: Gender and health equity. Washington, D. C.: PAHO.

García-Moreno, C., Jansen, H. A. F. M., Ellsberg, M., Heise, L., \& Watts, C. H. (2006). Prevalence of intimate partner violence: Findings from the Who multi-country study on women's health and domestic violence. Lancet, 368(7),1260-1269.

Gomes, I. C. (2005). Transmissão psíquica transgeracional e violência conjugal: um relato de caso. Boletim de Psicologia, 123(IV), 177188.

Heise, L. (1994). Gender-based Abuse: The Global Epidemic. Cadernos de Saúde Pública, 10(1), 135 - 145.

Klevens, J. (2001). Violencia física contra la mujer en Santa Fé de Bogotá: Prevalência y factores asociados. Revista Panamericana de Salud Pública, 9(2), 78-83.

Lamanno-Adamo, V. L. C. (1999). Violência doméstica: uma contribuição da Psicanálise. Ciência e Saúde Coletiva, 4(1),153159.

Melo, E. M., Melo, M. A. M., Pimenta, S. M. O., Lemos, S. M. A., Chaves, A. B., \& Pinto, L. M. N. (2007). Violência rompendo interações. As interações superando a violência. Revista Brasileira Saúde Materno Infantil, 7(1) ,89-98.

Menezes, T. C., Amorim, M. M. R., Santos, L. C., \& Faúndes, A. (2003). Violência física doméstica e gestação: resultados de um inquérito no puerpério. Revista Brasileira Ginecologia Obstetrícia, 25,309-316.

Messina, N., Grella, C. (2006). Childhood trauma and women's health outcomes in California Prision population. American Journal of Public Health, 96(2), 1842-1848.

Pan American Health Organization. (2003). La violencia baseada en el gênero: Un problema de salud pública y de derechos humanos. Washington, D. C.: Author.

Reichenheim, M. E., Moraes, C. L., \& Hasselmann, M. H. (2000). Equivalência semântica da versão em português do instrumento Abuse Assessment Screen para rastrear a violência contra a mulher grávida. Revista de Saúde Pública, 34(6), 610-16.

Reichenheim, M. E., Moraes, C. L., Szklo, A., Hasselmann, M. H., Souza, E. R., Lozana, J. A., \& Figueiredo, V. (2006), The magnitude of intime partner violence in Brazil: Portraits forom 15 capital cities and the Federal District. Cadernos de Saúde Pública, 22(2), 425-437.

Rivera-Rivera L., Lazcano-Ponce E., Salmerón-Castro J., SalazarMartinez E., Castro R., \& Hernández-Avila M. (2004). Prevalence and determinants male partner violence against mexican women: A population-based study. Salud Pública de México, 46, 113-122.

Saffiotti, H. I. B. (1999). Já se mete a colher em briga de marido e mulher. São Paulo em Perspectiva, 13(4), 82- 91. 
Saffiotti, H. I. B. (2005). Gênero e patriarcado: a necessidade da violência. Em M. C. Martin \& Sueli de Oliveira (Org.), Marcadas a ferro (pp. 35-74). Brasília: Secretaria Especial de Políticas para as Mulheres.

Schraiber, L. B., D’Oliveira, A. F. P. L., \& Couto, M. T. (2006) Violência e saúde: estudos científicos recentes. Revista de Saúde Pública, 40, 112-20.

Silva, I. V. (2003). Violência contra mulheres: a experiência de usuárias de um serviço de urgência e emergência de Salvador, Bahia, Brasil. Cadernos de Saúde Pública, 19(2), 5263-5272.

Velzeboer, M., Ellsberg, M., Clavel Arcas, C., \& García-Moreno, C. (2003). La violencia contra las mujeres: Respone el sector de la
salud/Violence against women: The health sector responds. Washington, D. C.: PAHO.

Vizcarra, M. B., Cortés, J., Bustos L., Alarcón, M., \& Muñoz, S. (2001). Violencia conyugal en la ciudad de Temuco. Un estudio de prevalencia y factores asociados. Revista Médica de Chile, 129(12), 1405-1412.

World Health Organization. (2001). Violence against women. Geneva: Author.

Recebido em 28/09/2007 Aceito em 09/06/2008

Endereço para correspondência :

Maria Arleide da Silva, Diretoria de Pesquisa/ Instituto de Medicina Integral Prof. Fernando Figueira. Rua dos Coelhos, 300, CEP: 50070-550, Boa Vista, Recife-PE, Brasil. E-mail: arleide@imip.org.br 